\title{
Photoconduction Properties in Aligned Assemblies of Colloidal CdSe/CdS Nanorods
}

\author{
Anna Persano, ${ }^{\dagger}$ Milena De Giorgi, ${ }^{\ddagger}$ Angela Fiore, ${ }^{\S}$ Roberto Cingolani, ${ }^{\ddagger}$ Liberato Manna, ${ }^{\S}$ Adriano Cola, ${ }^{\dagger}$ and \\ Roman Krahne ${ }^{\text {s.* }}$ \\ ${ }^{\dagger}$ Institute for Microelectronics and Microsystems (IMM-CNR), Unit of Lecce, Via Monteroni, I-73100 Lecce, Italy, ${ }^{\ddagger}$ National Nanotechnology Laboratoy of CNR-INFM, Via \\ Arnesano 16, 73100 Lecce, Italy, and §Italian Institute of Technology, Via Morego 30, 16163 Genova, Italy
}

ABSTRACT We report on photoconduction and optical properties of aligned assemblies of core-shell CdSe/ $\mathrm{CdS}$ nanorods prepared by a seeded growth approach. We fabricate oriented layers of nanorods by drop casting the nanorods from a solution on substrates with prepatterned, micrometer-spaced electrodes and obtain nanorod alignment due to the coffee stain effect. The photoconductivity of the nanorod layers can be improved significantly by an annealing process under vacuum conditions. The spectral response of the photocurrent shows distinct features that can be assigned to the electronic level structure of the core - shell nanorods and that relate well to the spectra obtained by absorption measurements. We study assemblies of nanorods oriented parallel and perpendicular to the applied electric field by the combined use of photocurrent and photoluminescence spectroscopy. We obtain consistent results which show that charge carrier separation and transport are more efficient for nanorods oriented parallel to the electric field. We also investigate the light polarization sensitivity of the photocurrent for the oriented nanorod layers and observe higher conductivity in the case of perpendicular polarization with respect to the long axis of the nanorods.

KEYWORDS: colloidal nanocrystals $\cdot$ nanorods $\cdot$ photoconductivity $\cdot$ selfassembly $\cdot$ coffee stain $\cdot$ seeded growth $\cdot$ CdSe $\cdot$ photoluminescence

*Address correspondence to roman.krahne@iit.it.

Received for review November 6, 2009 and accepted February 16, 2010.

Published online February 25, 2010. 10.1021/nn901575r

C 2010 American Chemical Society
$\mathrm{N}$

anorods are of great interest for both fundamental research and technical applications due to several unique electrical and optical properties, such as linearly polarized emission, ${ }^{1-3}$ exciton storage, ${ }^{4}$ electroluminescence, ${ }^{5}$ and efficient 1D electrical transport, ${ }^{6-9}$ which are determined by their anisotropic shape. In particular, core-shell nanorods fabricated by the seeded growth approach have several advantageous properties for optoelectronic applications. ${ }^{10,11}$ Due to their core-shell structure, such rods are highly luminescent and their emission wavelength can be tuned within certain limits not only by the shape of the rod but also even more efficiently by the size of the core. Moreover, the seeded growth approach yields a narrow distribution in nanorod length and diameter, which greatly facilitates their oriented assembly, ${ }^{12}$ and ordered nanorod assemblies over micrometer length scales have been reported by microfluidic ${ }^{13-18}$ and electric field-assisted assembly approaches. ${ }^{10,19-21}$ In order to obtain nanorod assemblies with a controlled orientation with respect to an external electric field, the electrodes can be fabricated prior to the nanorod deposition on a variety of substrates. ${ }^{10,18,22}$ Such devices with ordered assemblies of nanorods should exhibit novel properties and improved current transport ${ }^{23}$ compared to spherical nanocrystal arrays. ${ }^{24}$ Of particular interest for photoelectrical applications is the effect of the polarization of the incident light, as well as the impact of the applied electric field on exciton ionization, the charge transport through the inter-rod barrier, ${ }^{24}$ the carrier trapping and band-to-band recombination processes. ${ }^{4,10}$ All of these processes should depend strongly on the nanorod orientation.

In general, thin films of semiconductor nanocrystals represent a promising approach for active layers in photovoltaic devices and photosensors. To this end, multilayers of spherical nanocrystals, as well as randomly oriented nanorods, have been employed in vertical and planar electrode configurations. ${ }^{25-29}$ One obstacle toward electronic applications of nanocrystal arrays is their low conductivity due to the high tunnel barriers, which result from the organic ligands that passivate the nanocrystal surface. Successful approaches to overcome this obstacle consist of modifications of the nanocrystal surface chemistry after layer deposition, for example, by treatment with hydrazine or by thermal annealing processes. ${ }^{18,30,31}$

In this work, we present the photoconduction and optical properties of micrometer-scale aligned assemblies of core-shell $\mathrm{CdSe} / \mathrm{CdS}$ nanorods prepared 
by the seeded growth approach. ${ }^{10}$ The nanorods were synthesized by wet-chemical techniques starting from presynthesized CdSe seeds that were overcoated by a thick rod-shaped CdS shell. Coffee stain microfluidic dynamics were used to deposit layers of oriented nanorod assemblies ${ }^{32}$ on glass substrates on which micrometer-spaced interdigitated electrodes were fabricated in a previous step. The photocurrent was considerably enhanced by performing a moderate thermal annealing process under vacuum. The absorption and photocurrent spectra showed discrete features that reflected the electronic structure in the core and shell regions of the nanorods. We observed a significant dependence of the spectral photocurrent response on the nanorod orientation and an improved photoconductivity for nanorods with their long axis oriented parallel to the external electric field. The external electric field led to quenching of the photoluminescence $(\mathrm{PL})$, which increased with increasing voltage bias, and we found that this effect is more pronounced in assemblies with nanorods oriented parallel to the applied electric field. Finally, we investigated the polarization sensitivity of the spectral response and found negative values of the polarization anisotropy, $\rho=\left(\mathbf{I}_{\|}-\mathbf{I}_{\perp}\right) /\left(\mathbf{I}_{\|}+\mathbf{I}_{\perp}\right)$.

\section{RESULTS AND DISCUSSION}

One of the core-shell CdSe/CdS nanorod samples that we investigated in this work is shown in a transmission electron microscope image (TEM) in Figure 1a, together with an optical microscope image of the interdigitated electrode structure that we used for the photocurrent studies (Figure 1b).

After deposition of the nanorods onto the electrode structures, we used thermal annealing in order to improve the conductivity of the nanorod layers. Figure 1c shows the photocurrent of a nanorod layer when white light was switched on and off at fixed voltage bias, and we note that the photocurrent of such samples without any further treatment is very low. The photocurrent of a sample that had been annealed at $130{ }^{\circ} \mathrm{C}$ and was measured under vacuum is exhibited in Figure $1 \mathrm{~d}$. We found that the annealed samples showed an increase in conductivity by more than 1 order of magnitude. At the same time, the transient time constants increased also by more than 1 order of magnitude. In both cases, the conductivity decreased eventually to zero when the light was switched off; that is, sole photocurrent was measured. We note that the effects of the annealing were reversible and that sufficiently long storage under air (some hours) restored the photoelectrical properties that were measured prior to the annealing. The increase in photoconductivity and in transient time constants can be assigned to changes in the surface chemistry of the nanorods, which might also affect the average rod to rod distance in the dense layers. ${ }^{33}$ However, the trioctylphosphine
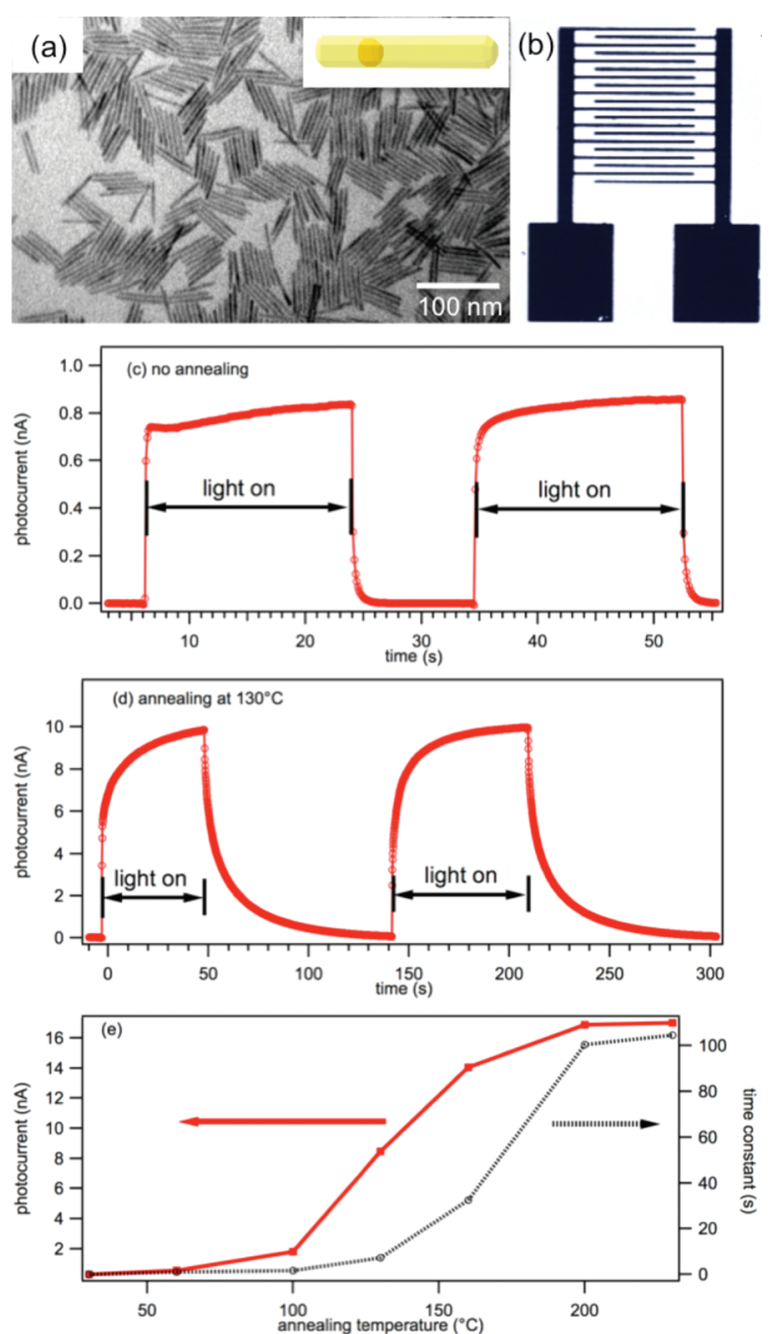

Figure 1. (a) Transmission electron microscope image of core-shell CdSe/CdS nanorods with $60 \mathrm{~nm}$ length and $4.5 \mathrm{~nm}$ diameter deposited on a carbon-coated grid. The inset illustrates the core-shell structure that resulted from the seeded growth approach. (b) Optical microscope image of an interdigitated electrode structure fabricated on a glass substrate. (c,d) Photocurrent of a dense layer of nanorods deposited onto the interdigitated electrodes, measured at a bias voltage of $V=84$ $V$ while switching the sample illumination by white light on and off. In (c), the nanorods were not annealed prior to the measurement, and in (d), the nanorods were annealed at 130 ${ }^{\circ} \mathrm{C}$ for $2 \mathrm{~h}$ under vacuum ( $p \sim 10^{-5} \mathrm{mbar}$ ). (e) Dependence of photocurrent and photocurrent decay time constants on the annealing temperature. The photocurrent was measured under vacuum at room temperature.

oxide (TOPO) ligands that stabilize the nanorod surface do not decompose at the comparatively low annealing temperatures that we used. We think that without annealing, some of the surface states might be passivated by water molecules due to humidity in the air and, therefore, cannot contribute to the charge transport between the rods. ${ }^{34}$ Annealing can remove the water molecules from the nanorod surface, and consequently, these surface trap states could contribute to the photoconductivity acting as intermediate states in the charge transport between rods. This mechanism would also explain why the 


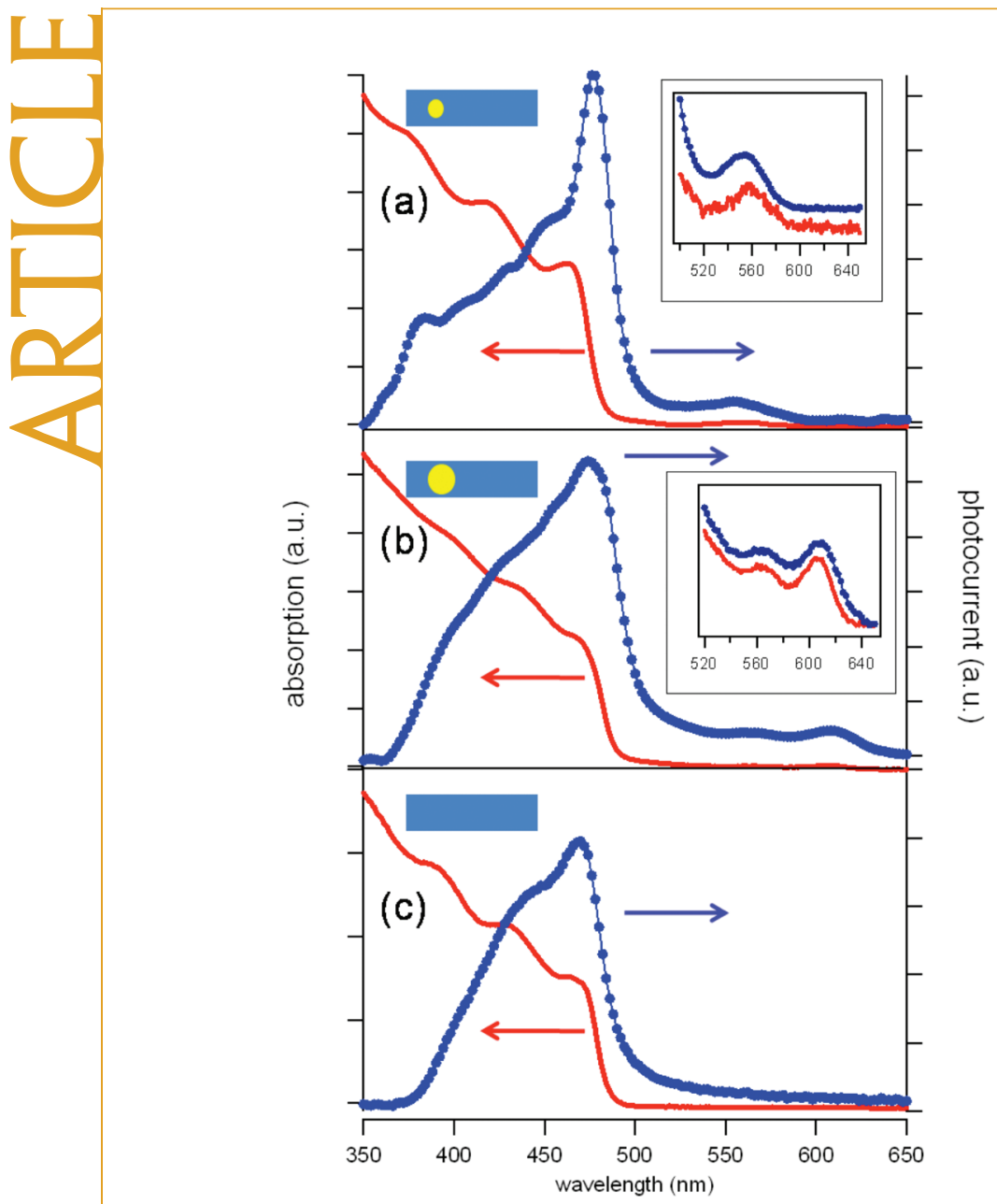

Figure 2. Photocurrent and absorption spectra of core-shell $\mathrm{CdSe} / \mathrm{CdS}$ nanorods with $60 \mathrm{~nm}$ length and different core sizes resulting in rod diameters of $4.5 \mathrm{~nm}$ (a) and $6 \mathrm{~nm}$ (b) and of CdS only (c) nanorods. The photocurrent was recorded at a bias voltage of $V=64 \mathrm{~V}$ under vacuum at room temperature from layers of nanorods deposited on interdigitated electrodes. The nanorod layers were annealed in order to obtain higher photoconductivity. The absorption was recorded from the nanorods in solution by a commercial spectrophotometer. The insets show absorption and photocurrent of the core on a magnified scale.

low conductivity and short transient times were recovered after storing the samples for some time under ambient conditions.

The photocurrent spectra of three interdigitated electrode structures covered with layers of different nanorod samples are displayed in Figure 2, together with the optical absorption of the same nanorod sample recorded in solution phase. The absorption spectra in Figure 2a,b show small peaks due to optical transitions in the CdSe core, and then a strong onset in absorption, in which several peaks can be resolved that correspond to optical transitions in the $\mathrm{CdS}$ shell of the nanorods. The insets of Figure $2 \mathrm{a}, \mathrm{b}$ show the wavelength range of the core absorption on a magnified scale, where we observe the absorption and photocurrent peaks of the rods with smaller core size at higher energy, as expected. Furthermore, for the larger core size (Figure 2b), we find a second peak originating from a transition into a higher energy level of the CdSe core. The photocurrent and the absorption spectra overlap well in the wavelength range displayed in the insets. However, in the ultraviolet (UV) range, we note a discrepancy between photocurrent and absorption spectra, as the absorption increases with increasing excitation energy, while the photocurrent of the nanorod layers decreases. The decrease in photocurrent at higher excitation energies could result from the more effective trapping of photogenerated charges in surface states, as reported in refs 35 and 36.

Figure $3 a, b$ shows polarized microscopy images of the aligned assemblies of nanorods that were formed in the outer fringe of the coffee stain, obtained with crossed polarizers and a $530 \mathrm{~nm}$ retardation plate at $45^{\circ}$ in the optical path. In these images, we clearly observe birefringence (different colors for different nanorod orientations) with the anisotropy axis oriented perpendicular (a) and parallel (b) to the electrodes (the electrodes appear as the black diagonal stripes in the images). We demonstrated in a previous work that the long axis of the nanorods coincides with the axis of anisotropy, ${ }^{32}$ which is also verified by the scanning electron microscopy (SEM) images of the nanorod layer in the region between the electrodes (Figure $3 c, d$ ).

Figure $4 a, b$ shows photoluminescence spectra of nanorod assemblies oriented parallel and perpendicular to the electric field for different bias voltage values. We find that the increase in voltage bias leads to an increased quenching of the photoluminescence, and that this effect is more pronounced for the rods oriented parallel to the electric field (see Figure 4c). This can be explained if we assume an anisotropic potential for the exciton confinement where the long dimension corresponds to the rod length, which is significantly larger than the exciton Bohr radius (about $5 \mathrm{~nm}$ for $\mathrm{CdS}$ ), whereas the short dimension corresponds to the rod diameter, which is of the same order as the exciton Bohr radius. Therefore, an external electric field that is oriented in the direction of the long axis of the nanorods is more efficient in separating the photogenerated electron-hole pairs.

The excitation wavelength of $405 \mathrm{~nm}$ leads to the photogeneration of carriers in the CdS shell. In order to observe the emission from the core, these carriers must be first captured from the CdS shell into the CdSe core. PL quenching arises because the applied electric field favors the separation of the photogenerated electrons and holes and thus prevents the carrier capture process into the core. We find that, if the bias voltage remains fixed (for example, at $V=120 \mathrm{~V}$ in Figure $4 \mathrm{~d}$ ), the quenching decreases as the excitation wavelength approaches the band gap of the nanorod core. In particular, the 
PL quenching induced by the external electric field is less effective for the longer excitation wavelengths at 488 and $512 \mathrm{~nm}$, which generate the carriers in the CdSe core. Similar results have been reported for InAs/GaAs self-assembled quantum dots. ${ }^{37}$ The orientation-dependent photocurrent curves are reported by the solid lines in Figure 4c, where we observe a significantly larger photocurrent for rods oriented parallel to the electric field. We note the good anticorrelation between the PL and the photocurrent intensity with respect to the bias voltage.

In order to gain more insight into the transport mechanism that governs the photogenerated charges in the oriented nanorod layers, we used the tunneling model of Leatherdale et al. ${ }^{35}$ to fit our orientation-dependent photocurrent data. This model was originally proposed for a close-packed solid of spherical dots and qualitatively reproduces the photocurrent by describing the probability of electron-hole pair separation as a function of the potential drop per dot (site-to-site potential). The extension of this model to a layer of oriented nanorods is motivated by the fact that it mainly relies on the tunneling process between nanoparticles that are separated by the potential barrier due to the or ganic capping, which is also the case for our oriented nanorod films. In this model, the tunneling current is expressed as

$I(v) \propto$

$$
\frac{\exp \left(\frac{-4 \sqrt{2} \hbar^{2} d}{3 m(e v-\gamma)}\left[\left(\frac{m \Phi}{\hbar^{2}}\right)^{3 / 2}-\left(\frac{m(\Phi+\gamma-e v}{\hbar^{2}}\right)^{3 / 2}\right]\right)}{1+\exp \left(-\frac{\gamma-e v}{a}\right)}
$$

Here $v$ is the voltage drop per quantum dot, $m$ is the rest mass of the electron, $e$ is the elementary charge, $\gamma$ is the energy cost between the initial and final states that arises from the energy required to overcome the Coulomb interaction of the photoexcited electron-hole pair and the interaction of each charge with its respective image charges. A phenomenological parameter $a$ describes the amount of tailing of the density of states into the energy gap, and $\Phi$ and $d$ are the height and the width of the tunnel barrier, respectively. The site-to-site potential can be evaluated by dividing the applied bias voltage by the number of rods that are needed to form a conductive path between the electrodes for the parallel and perpendicular configuration. Due to the aspect ratio of the nanorods, which is around 10, the site-to-site potential (i.e., the voltage drop on an individual nanorod) in the parallel configuration is 1 order of magnitude larger. This leads to the significantly higher photoconductivity in the parallel configuration. The fits of the current-voltage characteristics of the photocurrent yield a barrier height of
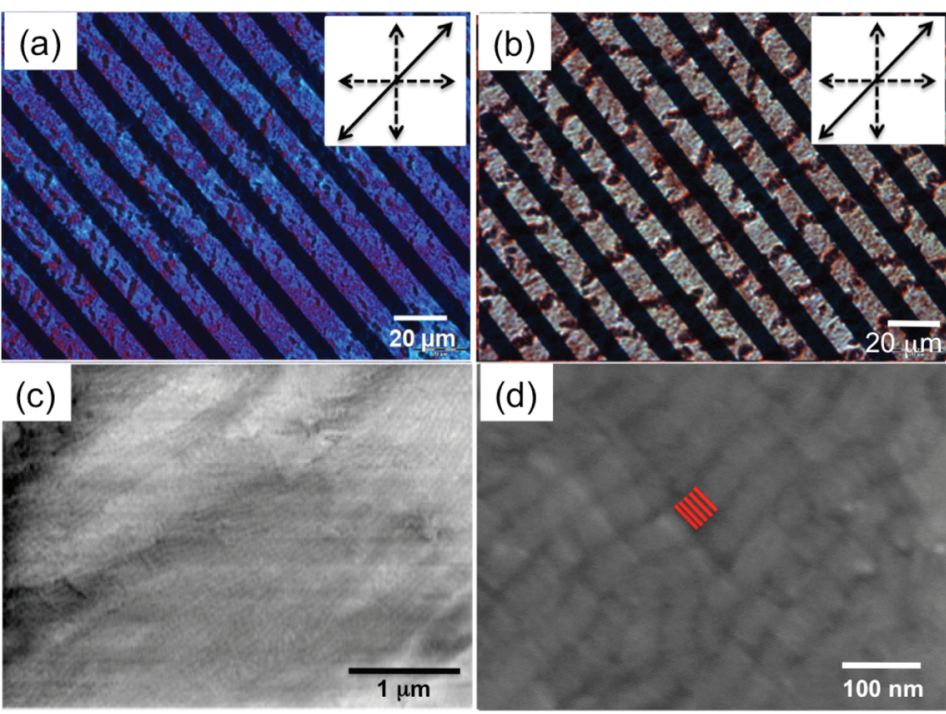

Figure 3. $(a, b)$ Polarized microscopy images of two devices with different alignment orientation, where the central region of the interdigitated electrodes is covered with a layer of nanorods. The images were obtained with crossed polarizers and a $530 \mathrm{~nm}$ retardation plate at $45^{\circ}$ in the optical path. In (a), the rods are oriented parallel to the electric field, in (b), they are perpendicular. The insets illustrate the orientations of the polarizers (dashed lines) and of the retardation plate (solid line) in the optical path. (c) Scanning electron microscopy images at different magnifications of an oriented layer of $\mathrm{CdSe} / \mathrm{CdS}$ nanorods with $60 \mathrm{~nm}$ length and $4.5 \mathrm{~nm}$ diameter in the region between the interdigitated electrodes. The orientation of the nanorods in the SEM images corresponds to the geometry in the polarized images displayed in (b), that is, with the long axis of the rods oriented perpendicular to the electric field. Some rods are highlighted in red as a guide to the eye.

1.2 and $0.3 \mathrm{eV}$ and a rod-to-rod distance of 0.8 and $1.1 \mathrm{~nm}$ for nanorods aligned parallel and perpendicular to the electric field, respectively. The fitting curves and the corresponding parameters are reported in the Supporting Information. The values for the barrier height differ significantly for the two configurations, whereas the parameter for the rodto-rod distance shows a much smaller variation and corresponds well to the length of the TOPO molecules on the nanorod surface. We propose that the difference in barrier height results from the difference in confinement length along and perpendicular to the nanorod axis. In the direction parallel to the nanorod axis, the confinement length is 1 order of magnitude larger than the Bohr radius, and the obtained barrier height of $1.2 \mathrm{eV}$ corresponds well to values assumed for $\mathrm{CdS}$ dots functionalized with TOPO. ${ }^{38}$ On the other hand, in the perpendicular direction to the nanorod axis, the confinement length and the Bohr radius are of the same order, and consequently the effective barrier height could be reduced.

Finally, we investigated photocurrent spectra of the oriented nanorod layers as a function of the polarization of the excitation light. Figure $5 a, b$ shows photocurrent spectra obtained by polarized excitation light at 0 and $90^{\circ}$ with respect to nanorod orientation, as illustrated in the inset. Figure $5 c, d$ shows the photocurrent amplitude at specific wavelengths versus the light polar- 


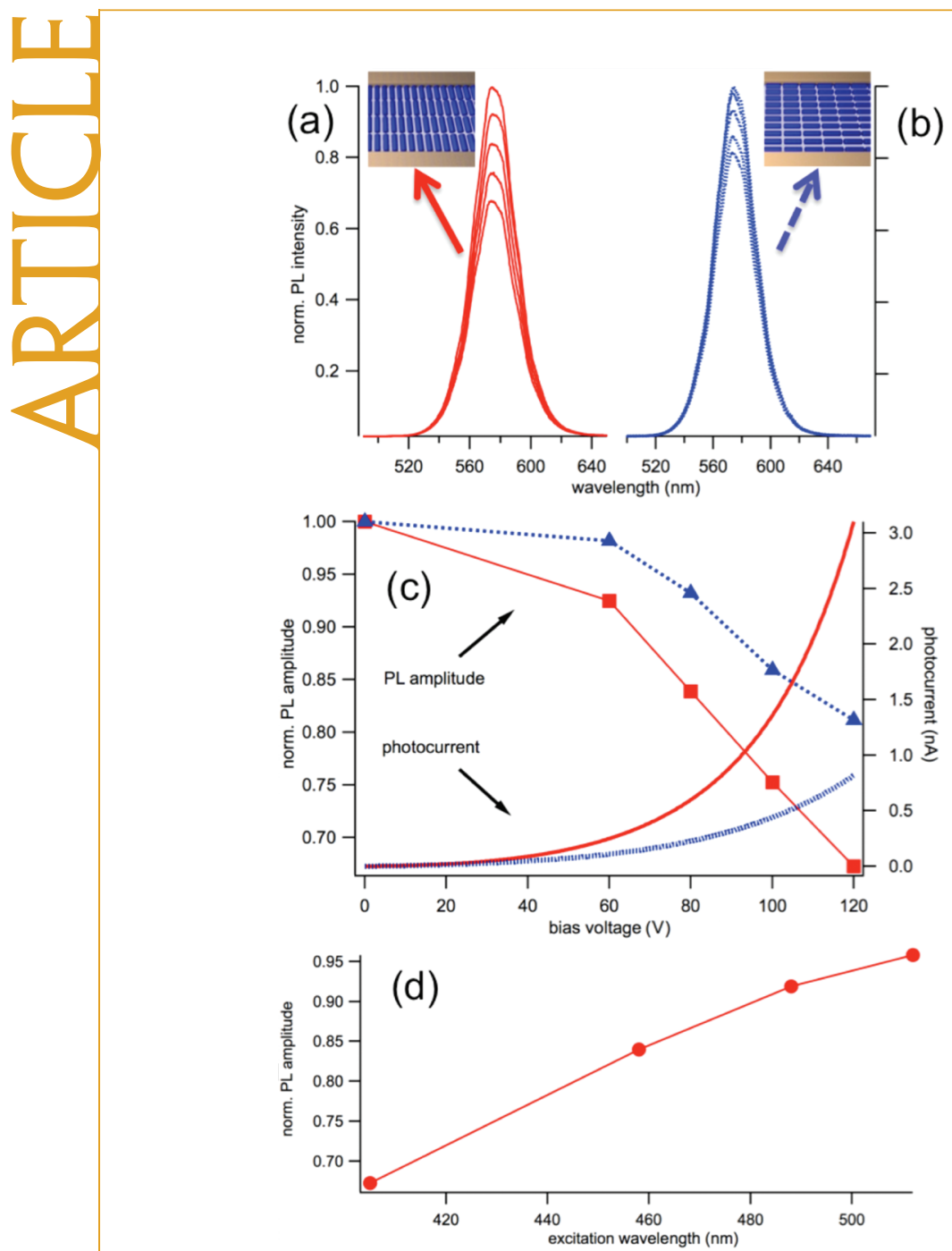

Figure 4. Optical and photoelectrical properties of layers of oriented nanorods with $4.5 \mathrm{~nm}$ diameter and $60 \mathrm{~nm}$ length. The data displayed by the solid red (dotted blue) lines correspond to nanorods oriented parallel (perpendicular) to the electric field. $(a, b)$ Photoluminescence (PL) spectra recorded from oriented nanorod layers at different bias voltages $(0,60,80,100,120 \mathrm{~V})$. The exciting laser wavelength was at $405 \mathrm{~nm}$, and the spectra were obtained at room temperature under air. (c) Normalized $\mathrm{PL}$ amplitude $\left(\mathrm{PL}_{\text {bias }} / \mathrm{PL}_{0}\right)$ (lines + markers), and the photocurrent intensity (lines) for different rod orientation as a function of bias voltage. (d) Normalized $\mathrm{PL}$ amplitude $\left(\mathrm{PL}_{\text {bias }} / \mathrm{PL}_{0}\right)$ of nanorods oriented parallel to the electric field for different excitation wavelengths at a bias voltage of $V=120 \mathrm{~V}$. ization angle for nanorods oriented parallel and perpendicular to the electric field, respectively. In both cases, the photocurrent intensity is larger when the light polarization is approximately perpendicular to the long axis of the nanorods. Polarization anisotropies have been determined using the expression $\rho=\left(\mathbf{I}_{\|}-\mathbf{I}_{\perp}\right) /\left(\mathbf{I}_{\|}+\mathbf{I}_{\perp}\right)$, where $\mathbf{I}_{\|}$and $\mathbf{I}_{\perp}$ are the intensities of the photocurrent with the excitation light polarized in the direction at $0^{\circ}$ (parallel) and $90^{\circ}$ (perpendicular) with respect to the long axis of the nanorods, respectively. We find that the polarization anisotropy $\rho$ is always negative and that $|\rho|$ is larger when the rods are oriented perpendicular to the electric field. This result is at first glance surprising because the absorption efficiency (and thus the photogeneration of the charges) has been found to be larger for light polarized parallel to the long axis of nanorods. ${ }^{39}$ However, we could understand this peculiar behavior if we consider that the competing process to photoconductivity (the radiative recombination of the photogenerated charges) is more effective in the direction parallel to the rod axis, which is manifested by polarized emission parallel to the long axis of the nanorods. ${ }^{2,3,10}$

In conclusion, we have studied the photoconduction and optical properties of aligned assemblies of core-shell CdSe/CdS nanorods. The photoconductivity of our samples could be significantly improved by thermal annealing under vacuum. The dominant energy range for photogeneration of charges in nanorod films could be extracted from their photocurrent spectra. Photocurrent and photoluminescence spectra consistently showed that the external electric field is more efficient in the separation of photogenerated charges for layers in which the nanorods are oriented parallel, rather than perpendicular, to the electric field. Finally, photocurrent spectra recorded as a function of the polarization of the excitation light yielded negative values of photocurrent anisotropy. We believe that the orientation-dependent optical and photoelectrical properties of aligned nanorod arrays reported in this paper can give valuable information for the design of photovoltaic devices, electro-modulators, and photosensors.

\section{EXPERIMENTAL DETAILS}

Core-shell CdSe/CdS nanorods with nearly monodisperse size distribution were prepared using the seeded growth approach described in ref 10 . We discuss here the photoconductive properties of two representative nanorod samples of $\mathrm{CdSe} / \mathrm{CdS}$ nanorods with $60 \mathrm{~nm}$ length and different core size resulting in diameters of 4.5 and $6 \mathrm{~nm}$. Nanorods with $40 \mathrm{~nm}$ length and $5 \mathrm{~nm}$ diameter consisting solely of $\mathrm{CdS}$ material were fabricated by the same synthesis method using a $\mathrm{CdS}$ seed. The nanorods were drop casted from solution onto glass substrates that were patterned with interdigitated gold electrodes in a previous fabrication step, and the solution was allowed to evaporate under ambient conditions.

The interdigitated electrodes on the glass substrates were fabricated by standard optical lithography followed by metal evaporation of $5 \mathrm{~nm} \mathrm{Cr}$ and $50 \mathrm{~nm} \mathrm{Au}$. Due to the peculiar solvent evaporation and fluid convection dynamics, usually referred to as the "coffee stain" effect, ${ }^{17,32,40,41}$ the nanorods present in solution were transported to the pinned contact line and started to form large-scale ordered superstructures that are reminiscent of nematic/smectic liquid crystal phases upon evaporation of the solvent. In detail, the nanorods formed tracks in which they assembled side by side over length scales of tens of micrometers. These tracks were oriented mainly perpendicular to the film border, and therefore, the majority of the individual rods were oriented parallel to the edge of the coffee stain. We used $15 \mu \mathrm{L}$ of nanorod solution with a nanorod concentration of $5 \times 10^{-7} \mathrm{M}$ that resulted in a coffee stain of approximately 1 $\mathrm{cm}$ diameter on the glass slides. For our measurements on ori- 

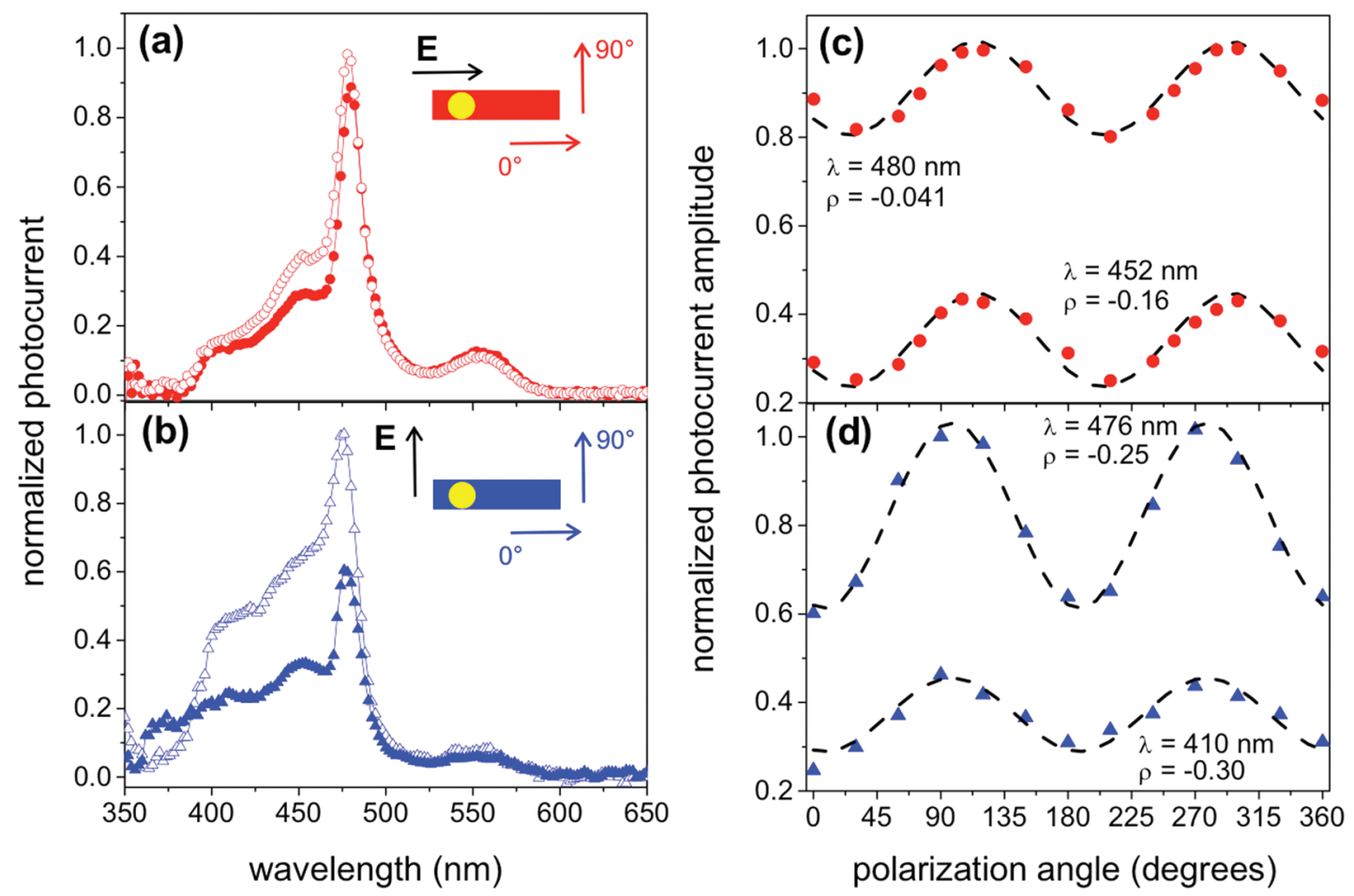

Figure 5. Normalized photocurrent spectra at voltage bias of $V=64 \mathrm{~V}$ with excitation light polarized at $0^{\circ}$ (closed symbols) and $90^{\circ}$ (open symbols) with respect to the nanorods parallel (a) and perpendicular (b) to the electric field. (c,d) Dependence of the photocurrent at specific wavelengths on the polarization angle for the nanorods parallel (c) and perpendicular (d) to the electric field. The sinusoidal dashed lines are a guide to the eye and show that the photocurrent polarization has a period of $\pi$. The values of the polarization anisotropy $\rho=\left(I_{\|}-I_{\perp}\right) /\left(I_{\|}+I_{\perp}\right)$ are also reported, where $I_{\|}$and $I_{\perp}$ are the intensities of the photocurrent with the excitation polarized at 0 and $90^{\circ}$, respectively, with respect to the direction of the long nanorod axis.

ented nanorod assemblies, we selected the interdigitated electrode structures near the border of the stain such that the electric field direction was either parallel or perpendicular oriented with respect to the long axis of the nanorods. Electrode fingers having width and spacing of 10 and $8 \mu \mathrm{m}$, respectively, were used for measurements, and the applied voltages ranged from 0 to $120 \mathrm{~V}$.

All of the electrical measurements were carried out in an electromagnetically shielded probe station at room temperature and under vacuum $\left(p \sim 10^{-5} \mathrm{mbar}\right)$. A Yokogawa 7651 dc source was used as voltage supply, and the current was measured with a DL 1211 current amplifier. This setup allowed us to record transients during switch on/off experiments of the sample illumination by sampling the current at the rate of $(40 \mathrm{~ms})^{-1}$.

For the spectral photocurrent experiments, a $175 \mathrm{~W}$ xenon lamp in combination with a $0.11 \mathrm{~m}$ monochromator was used as the excitation source. The light intensity was monitored with a calibrated Si photodiode in order to provide the spectral curve used to normalize the device response. The spot size was $\sim 50$ $\mu \mathrm{m}$ in diameter with an illumination intensity of $\sim 142 \mathrm{nW}$ at 480 $\mathrm{nm}$. PL experiments were performed with a confocal system (Olympus FV1000) in epilayer configuration. The samples were excited by diode lasers at $405,458,488$, and $512 \mathrm{~nm}$ through a $40 \times$ objective lens with a numerical aperture of NA $=0.8$. The emission from areas with $10 \mu \mathrm{m}$ diameter, which were selected with the confocal system, was detected by a $0.32 \mathrm{~m}$ monochromator equipped with a nitrogen-cooled CCD camera (Jobin Ivon) that was coupled to the confocal head (the spectral resolution of the whole system is $0.5 \mathrm{~nm}$ ). All PL measurements were performed in air at room temperature.

Supporting Information Available: Fitting curves and the corresponding parameters that we obtained from applying the tunneling model to the current voltage characteristics of the oriented nanorod layers are reported. This material is available free of charge via the Internet at http://pubs.acs.org.

\section{REFERENCES AND NOTES}

1. Peng, X. G.; Manna, L.; Yang, W. D.; Wickham, J.; Scher, E.; Kadavanich, A.; Alivisatos, A. P. Shape Control of CdSe Nanocrystals. Nature 2000, 404, 59-61.

2. Hu, J. T.; Li, L. S.; Yang, W. D.; Manna, L.; Wang, L. W.; Alivisatos, A. P. Linearly Polarized Emission from Colloidal Semiconductor Quantum Rods. Science 2001, 292, 2060-2063.

3. Talapin, D. V.; Koeppe, R.; Gotzinger, S.; Kornowski, A.; Lupton, J. M.; Rogach, A. L.; Benson, O.; Feldmann, J.; Weller, H. Highly Emissive Colloidal CdSe/CdS Heterostructures of Mixed Dimensionality. Nano Lett. 2003, 3, 1677-1681.

4. Kraus, R. M.; Lagoudakis, P. G.; Rogach, A. L.; Talapin, D. V.; Weller, H.; Lupton, J. M.; Feldmann, J. Room-Temperature Exciton Storage in Elongated Semiconductor Nanocrystals. Phys. Rev. Lett. 2007, 98, 017401.

5. Gudiksen, M. S.; Maher, K. N.; Ouyang, L.; Park, H. Electroluminescence from a Single-Nanocrystal Transistor. Nano Lett. 2005, 5, 2257-2261.

6. Steiner, D.; Katz, D.; Millo, O.; Aharoni, A.; Kan, S.; Mokari, T.; Banin, U. Zero-Dimensional and Quasi One-Dimensional Effects in Semiconductor Nanorods. Nano Lett. 2004, 4, 1073-1077.

7. Steiner, D.; Dorfs, D.; Banin, U.; Della Sala, F.; Manna, L.; Millo, O. Determination of Band Offsets in Heterostructured Colloidal Nanorods Using Scanning Tunneling Spectroscopy. Nano Lett. 2008, 8, 2954-2958.

8. Trudeau, P. E.; Sheldon, M.; Altoe, V.; Alivisatos, A. P. Electrical Contacts to Individual Colloidal Semiconductor Nanorods. Nano Lett. 2008, 8, 1936-1939.

9. Steinberg, H.; Lilach, Y.; Salant, A.; Wolf, O.; Faust, A.; Millo, O.; Banin, U. Anomalous Temperature Dependent Transport through Single Colloidal Nanorods Strongly Coupled to Metallic Leads. Nano Lett. 2009, 9 , 3671-3675. 
10. Carbone, L.; Nobile, C.; De Giorgi, M.; Della Sala, F.; Morello, G.; Pompa, P.; Hytch, M.; Snoeck, E.; Fiore, A.; Franchini, I. R.; Nadasan, M.; Silvestre, A. F.; Chiodo, L.; Kudera, S.; Cingolani, R.; Krahne, R.; Manna, L. Synthesis and Micrometer-Scale Assembly of Colloidal CdSe/CdS Nanorods Prepared by a Seeded Growth Approach. Nano Lett. 2007, 7, 2942-2950.

11. Talapin, D. V.; Nelson, J. H.; Shevchenko, E. V.; Aloni, S.; Sadtler, B.; Alivisatos, A. P. Seeded Growth of Highly Luminescent $\mathrm{CdSe} / \mathrm{CdS}$ Nanoheterostructures with Rod and Tetrapod Morphologies. Nano Lett. 2007, 7, 2951-2959.

12. Baranov, D.; Fiore, A.; van Huis, M.; Giannini, C.; Falqui, A.; Lafont, U.; Zandbergen, H.; Zanella, M.; Cingolani, R.; Manna, L. Assembly of Colloidal Semiconductor Nanorods in Solution by Depletion Attraction. Nano Lett. 2010, 10, 743-749.

13. Jana, N. R. Shape Effect in Nanoparticle Self-Assembly. Angew. Chem., Int. Ed. 2004, 43, 1536-1540.

14. Ghezelbash, A.; Koo, B.; Korgel, B. A. Self-Assembled Stripe Patterns of CdS Nanorods. Nano Lett. 2006, 6, 1832-1836.

15. Li, L. S.; Alivisatos, A. P. Semiconductor Nanorod Liquid Crystals and Their Assembly on a Substrate. Adv. Mater. 2003, 15, 408-411.

16. He, J.; Zhang, Q.; Gupta, S.; Emrick, T.; Russell, T. R.; Thiyagarajan, P. Drying Droplets: A Window into the Behavior of Nanorods at Interfaces. Small 2007, 3, 1214 1217.

17. Querner, C.; Fischbein, M. D.; Heiney, P. A.; Drndic, M. Millimeter-Scale Assembly of CdSe Nanorods into Smectic Superstructures by Solvent Drying Kinetics. Adv. Mater. 2008, 20, 2308-2314.

18. Steiner, D.; Azulay, D.; Aharoni, A.; Salant, A.; Banin, U.; Millo, O. Photoconductivity in Aligned CdSe Nanorod Arrays. Phys. Rev. B 2009, 80, 195308.

19. Hu, Z.; Fischbein, M. D.; Querner, C.; Drndić, M. Electric Field Driven Accumulation and Alignement of CdSe and CdTe Nanorods in Nanoscale Devices. Nano Lett. 2006, 6, 2585-2591.

20. Harnack, O.; Pacholski, C.; Weller, H.; Yasuda, A.; Wessels, J. M. Rectifying Behavior of Electrically Aligned $\mathrm{ZnO}$ Nanorods. Nano Lett. 2003, 3, 1097-1101.

21. Ryan, K. M.; Mastroianni, A.; Stancil, K. A.; Liu, H. T.; Alivisatos, A. P. Electric-Field-Assisted Assembly of Perpendicularly Oriented Nanorod Superlattices. Nano Lett. 2006, 6, 1479-1482.

22. Nobile, C.; Fonoberov, V. A.; Kudera, S.; Della Torre, A.; Ruffino, A.; Chilla, G.; Kipp, T.; Heitmann, D.; Manna, L.; Cingolani, R.; Balandin, A. A.; Krahne, R. Confined Optical Phonon Modes in Aligned Nanorod Arrays Detected by Resonant Inelastic Light Scattering. Nano Lett. 2007, 7, 476-479.

23. Romero, H. E.; Calusine, G.; Drndic, M. Current Oscillations, Switching, and Hysteresis in CdSe Nanorod Superlattices. Phys. Rev. B 2005, 72, 235401.

24. Steiner, D.; Azulay, D.; Aharoni, A.; Salant, A.; Banin, U.; Millo, O. Electronic Structure and Self-Assembly of CrossLinked Semiconductor Nanocrystal Arrays. Nanotechnol. 2008, 19, 065201

25. Murray, C. B.; Kagan, C. R.; Bawendi, M. G. Synthesis and Characterization of Monodisperse Nanocrystals and ClosePacked Nanocrystal Assemblies. Annu. Rev. Mater. Sci. 2000, 30, 545-610.

26. Ginger, D. S.; Greenham, N. C. Charge Injection and Transport in Films of CdSe Nanocrystals. J. Appl. Phys. 2000, 87, 1361-1368.

27. Huynh, W. U.; Dittmer, J. J.; Teclemariam, N.; Milliron, D. J.; Alivisatos, A. P.; Barnham, K. W. J. Charge Transport in Hybrid Nanorod-Polymer Composite Photovoltaic Cells. Phys. Rev. B 2003, 67, 115326.

28. Gur, I.; Fromer, N. A.; Geier, M. L.; Alivisatos, A. P. Air-Stable All-Inorganic Nanocrystal Solar Cells Processed from Solution. Science 2005, 310, 462-465.
29. Persano, A.; Leo, G.; Manna, L.; Cola, A. Charge Carrier Transport in Thin Films of Colloidal CdSe Quantum Rods. J. Appl. Phys. 2008, 104, 074306.

30. Yu, D.; Wang, C. J.; Guyot-Sionnest, P. n-Type Conducting CdSe Nanocrystal Solids. Science 2003, 300, 1277-1280.

31. Talapin, D. V.; Murray, C. B. PbSe Nanocrystal Solids for $n$ and $\mathrm{p}$-Channel Thin Film Field-Effect Transistors. Science 2005, 310, 86-89.

32. Nobile, C.; Carbone, L.; Fiore, A.; Cingolani, R.; Manna, L.; Krahne, R. Self-Assembly of Highly Fluorescent Semiconductor Nanorods into Large Scale Smectic Liquid Crystal Structures by Coffee Stain Evaporation Dynamics. J. Phys.: Condens. Matter 2009, 21, 264013.

33. Drndic, M.; Jarosz, M. V.; Morgan, N. Y.; Kastner, M. A.; Bawendi, M. G. Transport Properties of Annealed CdSe Colloidal Nanocrystal Solids. J. Appl. Phys. 2002, 92, 74987503.

34. Alperson, B.; Rubinstein, I.; Hodes, G. Identification of Surface States on Individual CdSe Quantum, Dots by Room-Temperature Conductance Spectroscopy. Phys. Rev. B 2001, 6308, 081303.

35. Leatherdale, C. A.; Kagan, C. R.; Morgan, N. Y.; Empedocles, S. A.; Kastner, M. A.; Bawendi, M. G. Photoconductivity in Cdse Quantum Dot Solids. Phys. Rev. B 2000, 62, 2669-2680.

36. Cretí, A.; Anni, M.; Zavelani-Rossi, M.; Lanzani, G.; Leo, G.; Della Sala, F.; Manna, L.; Lomascolo, M. Ultrafast Carrier Dynamics in Core and Core/Shell CdSe Quantum Rods: Role of the Surface and Interface Defects. Phys. Rev. B 2005, 72, 125346.

37. Fry, P. W.; Finley, J. J.; Wilson, L. R.; Lemaitre, A.; Mowbray, D. J.; Skolnick, M. S.; Hopkinson, M.; Hill, G.; Clark, J. C. Electric-Field-Dependent Carrier Capture and Escape in Self-Assembled InAs/GaAs Quantum Dots. Appl. Phys. Lett. 2000, 77, 4344-4346.

38. Pellegrini, G.; Mattei, G.; Mazzoldi, P. Finite Depth Square Well Model: Applicability and Limitations. J. Appl. Phys. 2005, 97, 073706.

39. Lutich, A.; Carbone, L.; Volchek, S.; Yakovtseva, V.; Sokol, V.; Manna, L.; Gaponenko, S. Macroscale Alignment of CdSe/ CdS Nanorods by Porous Anodic Alumina Templates. Phys. Status Solidi Lett. 2009, 3, 151-153.

40. Deegan, R. D.; Bakajin, O.; Dupont, T. F.; Huber, G.; Nagel, S. R.; Witten, T. A. Capillary Flow as the Cause of Ring Stains from Dried Liquid Drops. Nature 1997, 389, 827-829.

41. Jung, J.-Y.; Kim, Y. W.; Yoo, J. Y. Behavior of Particles in an Evaporating Didisperse Colloid Droplet on a Hydrophilic Surface. Anal. Chem. 2009, 81, 8256-8259. 